\title{
Proyectos hidráulicos, ambientalismos y re-escalamiento territorial: la disputa en torno a la construcción del proyecto Paraná medio en el proceso de neoliberalización de Argentina, 1995-19971
}

\author{
Gisela Ariana Rausch²
}

\begin{abstract}
RESUMEN
Se analiza el conflicto territorial-ambiental en Santa Fe y Entre Ríos, surgido con motivo del proyecto hidroeléctrico Paraná Medio durante la década de 1990, y su resolución a favor de la coalición opositora. Se sostiene que dicho triunfo se debió, en gran parte, a condiciones específicas del contexto mundial y nacional, pero también a características que adoptaron las organizaciones ambientalistas locales, inusuales hasta ese momento en Argentina. Utilizando una metodología cualitativa, sustentada en fuentes documentales y entrevistas abiertas, se indagó en la producción discursiva en torno al proyecto en el contexto de dos procesos impulsados en Argentina en dicha década: la instalación del neoliberalismo como ideología político-económica hegemónica, y la emergencia del "ambientalismo" como marco de referencia y fuerza social condensadora de reclamos sociales de carácter territorial.
\end{abstract}

Palabras claves: Proyectos hidráulicos, neoliberalismo, ambientalismos, conflictos territoriales.

\begin{abstract}
It is discussed the territorial-environmental conflict in Santa Fe and Entre Ríos, emerged on the occasion of the Paraná Medio hydroelectric project during the 1990s and its resolution in favor of the opposition coalition. It is argued that this triumph was due, in large part, to specific conditions of global and national context, but also to unusual characteristics that have adopted local environmental organizations so far in Argentina. Using a qualitative methodology, based on interviews and documentary sources, we inquire into the discursive production around the project in the context of two processes driven in Argentina in that decade: the installation of neo-liberalism as hegemonic political and economic ideology, and the emergence of environmentalism as a frame of reference and condensing social force of territorial claims.
\end{abstract}

Key words: Hydraulic projects, neoliberalism, environmentalism, territorial conflicts. 
Durante buena parte del siglo XX, los proyectos hidráulicos han sido considerados símbolos de grandeza nacional y progreso económico. Estrechamente vinculados al ideario desarrollista de posguerra, se convirtieron en poderosos instrumentos de territorialización estatal, siendo promovidos como piezas relevantes en las políticas de modernización nacional y desarrollo regional, que, en los países de economías centrales, supusieron procesos de industrialización, urbanización y construcción de infraestructura.

Los estudios sobre grandes represas constituyen un vasto campo que involucra múltiples disciplinas. Entre las referencias más relevantes que han contribuido a nuestra investigación, destacamos los trabajos que abordan las vinculaciones entre poder y grandes proyectos hidráulicos ${ }^{3}$ (GPH) como la ya clásica obra de Karl Wittfogel (1957), con su propuesta teórica de las sociedades hidráulicas para el análisis de las relaciones entre irrigación y poder totalitario en China; David Worster (1985 y 2011), quien indagó en las relaciones entre las tecno-burocracias estatales y la expansión capitalista mediante el control del agua. Gustavo Lins Ribeiro (1994), quien tomando como caso de estudio la represa argentina Yacyretá, analizó los procesos de transnacionalización capitalista en América Latina. También Patrick Mc Cully (2001) ha dedicado buena parte de su obra a la crítica de las grandes represas como infraestructuras al servicio del poder en la segunda posguerra. Más recientemente, desde la perspectiva de la Ecología Política, María Kaika (2005, 2006a, 2006b, 2009), Erik Swyngedouw (2015) y Karen Bakker (2010) han abordado los paradigmas hidráulicos en general, y la problemática de las represas en particular, como símbolos de modernización de los Estados nacionales.

En el ámbito nacional, pueden mencionarse los estudios realizados en el Centro de Estudios Urbanos y Regionales (CEUR) por Rofman, Brunstein, Laurelli y Vidal (1987) acerca de los grandes proyectos en general y los proyectos hidráulicos en particular. Desde la Antropología, Catullo (1997 y 2008) y Catullo y Coun (2002) han indagado en los procesos de relocalización poblacional y los efectos sociales de las grandes represas. También Azcoitía y Nuñez (2014), desde la Historia, han analizado los debates en torno a las ideas de desarrollo vinculadas a la construcción de represas en la región Comahue.

Durante el siglo XX, los GPH han destacado tanto por la magnitud de las inversiones que requirieron (de ahí, en parte, su denominación de elefantes blancos) como por las dimensiones que posteriormente cobraron sus impactos socioambientales (WCD, 2000 y AIDA, 2009; Fernández y Carrillo, 2010). Por ambos motivos, hacia la década de 1980, los GPH comenzaron a ser cuestionados, convirtiéndose en foco de rechazo social (Mc Cully (2001), y hacia la década de 1990, en objetos de revisión por parte de los organismos internacionales que durante el siglo XX habían sido sus principales impulsores (WCD, 2000).

Las grandes represas traen aparejadas drásticas transformaciones territorio-ambientales. Así, por ejemplo, una obra paradigmática concretada en Argentina por la última dictadura militar, fue la represa binacional Salto Grande (1979), cuya construcción implicó inundar la ciudad de Federación (en Argentina), Belén y Constitución (en Uruguay), con la consecuente relocalización de las

Se considera Gran Proyecto Hidráulico a aquel que involucra una Gran Represa según la definición de la Comisión Internacional de Grandes Represas (ICOLD, en inglés), es decir, aquella cuya altura mínima es de 15 metros o con embalse mayor a 3 millones de m3 (Fernández, 2010: 16). 
respectivas poblaciones. También ha sido relevante el caso de la represa Yacyretá (1994), tanto por las dimensiones que cobraron los hechos de corrupción (Mc Cully, 2001:160-163) como por la magnitud de los efectos en la biodiversidad y las poblaciones locales (Lins Ribeiro, 1994). A su vez, el proyecto Corpus Christi, en Misiones, fue suspendido como resultado de un plebiscito llevado a cabo en 1996 (Suárez Montórfano, 2008).

En el año 1996, y en el contexto de los conflictos por Yacyretá, se presentaba el PHPM. El hecho suscitó inmediatamente acaloradas discusiones, principalmente en Santa Fe y Entre Ríos, conformándose una arena de debates y luchas entre diferentes intereses, que se alinearon en coaliciones a favor y en contra. En la contienda, cobraron relevancia los discursos ambientalistas, que provenían principalmente de las ONGs locales, y le proporcionaron legitimidad y mayor difusión al reclamo de los pobladores locales que iban a ser afectados por el embalse. Las acciones conjuntas entre éstos y las asociaciones ambientalistas, conformando una coalición anti-represa con características inusuales para la época, que culminaron en la confección de una ley provincial sancionada en Entre Ríos, prohibiendo el represamiento de los ríos Paraná y Uruguay en el tramo de dicha provincia. Esta ley dio punto final a la tentativa de llevar a cabo el proyecto hidroeléctrico.

Una serie de consideraciones han delineado la investigación: 1) por las características de la coalición opositora, el conflicto suscitado puede considerarse un caso de transición entre las luchas ambientalistas más tradicionales y el llamado giro ecoterritorial ${ }^{4}$ en los movimientos sociales de América Latina, propios del siglo XXI (Svampa, 2011 y 2012); 2) puede decirse que dicho conflicto constituyó una de las primeras manifestaciones, en Argentina, de resistencia social en defensa del territorio por cuestiones ambientales, al tiempo que el modus operandi de las asociaciones ambientalistas en conjunto con los sectores sociales locales, casi no registra antecedentes en el país; 3) junto con el proyecto hidroeléctrico Corpus Christi, el PHPM fue, en Argentina, una de las únicas grandes obras de infraestructura que, durante la década neoliberal, fueron suspendidas ante la contestación social. Dicha suspensión estuvo vinculada estrechamente a condiciones históricas específicas que se dieron a nivel internacional, nacional y provincial (en Entre Ríos); y 4) en los discursos de la coalición opositora se reconocen elementos argumentales y valorativos que hoy forman parte del Ilamado pensamiento latinoamericano del siglo XXI, que buscan alternativas al desarrollo hegemónico (Grupo Permanente de Trabajo sobre Alternativas al Desarrollo, 2012; Svampa y Viale, 2014; Gudynas, 2015).

Se sostiene que los GPH constituyen nodos de convergencia multiescalar de procesos humanos y no humanos, de elementos materiales y discursivos, mecánicos y orgánicos, estrechamente vinculados a los procesos políticos y sociales pero también a las aspiraciones de cambio social y a las concepciones acerca de lo que se ha llamado naturaleza (Swyngedouw, 2015:5). Atendiendo a ello, el artículo tiene como objetivo más general abordar históricamente el conflicto social motivado por el PHPM, colocando el énfasis en la dimensión discursiva (lo que es dicho) en torno a la construcción de los GPH, en su contexto político-económico y cultural de producción. En particular, se analizan los discursos en torno al PHPM en el contexto de dos procesos históricos: 1) la instalación del neoliberalismo como ideología político-económica hegemónica en la década de 1990 y 2) la emergencia del ambientalismo como marco de referencia y fuerza social condensado-

El término utilizado por Svampa (2012:190) hace referencia a las luchas socioambientales en América Latina que en el presente siglo han conformado "un lenguaje común de valoración sobre la territorialidad", articulando el comunitarismo indígena con los discursos ambientalistas. 
ra de reclamos sociales de carácter territorial, que se consolidaría en el siguiente siglo (Svampa, 2010, 2011 y 2012; Svampa y Viale, 2014).

La indagación puede ubicarse en un campo interdisciplinar que, desde una perspectiva histórica, articula las contribuciones de la Geografía Humana y Cultural, la Ecología Política y los estudios urbano-territoriales en general. Se adoptó una metodología cualitativa sustentada en fuentes documentales y entrevistas abiertas a algunos actores claves. En particular, se han analizado documentos producidos por diversos organismos y administraciones públicas, artículos de la prensa local, regional y nacional correspondiente al período comprendido entre 1990 y 1997 , publicaciones varias de las asociaciones ambientalistas que participaron en el conflicto y documentación técnica del proyecto en cuestión.

La primera parte del artículo desarrolla una breve sinopsis histórica del PHPM en su etapa de formulación anterior a la década de 1990. En la segunda parte, se describen y analizan las condiciones contextuales en que tuvo lugar el proyecto, durante la década de 1990, atendiendo al proceso de implementación de políticas neoliberales en América Latina y en Argentina en particular, como así también a la conformación del Mercado Común del Sur (MERCOSUR) que supuso una nueva escala territorial de operaciones económicas. La tercera parte aborda el conflicto socioambiental, analizando los discursos de las coaliciones a favor y en contra del proyecto, junto a las estrategias desplegadas por la oposición para lograr su suspensión.

Finalmente se presentan algunas reflexiones sobre el conflicto en cuestión, destacando la condición de los proyectos hidráulicos de constituirse en nodos de convergencia de determinados intereses (políticos, económicos, sociales) y controversias, conformando lo que Swyngedouw (2015:5) ha denominado una "constelación hidrosocial". Y en particular, la consideración del PHPM como caso de transición entre dos modos de concebir el territorio, los recursos naturales y las luchas ambientales.

\section{El Proyecto Hidroeléctrico Paraná Medio en el proyecto estatal: breve sinopsis histórica entre 1958 y 1986}

El PHPM se presentó por primera vez en 1958 junto con la represa Yacyretá, aunque no comenzó a desarrollarse hasta mediados de la década de 1970, enmarcado en el "Plan Nacional de Desarrollo y Seguridad 1971-1975". Ambas represas formaban parte de un programa de aprovechamiento hidroeléctrico del río Paraná, que incluía la generación de energía y diversas obras complementarias (ferroviales y de control de crecidas, entre otras) que serían llevadas a cabo por la empresa estatal Agua y Energía. En el caso del PHPM, la financiación provendría de una empresa soviética (Technopromexport) que había construido la represa de Asuan (Egipto). Este convenio tenía lugar en el marco de las relaciones comerciales que el gobierno argentino mantuvo con el gobierno de la URSS hasta principios de la década de 1980.

El proyecto en cuestión se ubicaba en el tramo medio del río Paraná, abarcando tres provincias argentinas: Corrientes, Santa Fe y Entre Ríos, pertenecientes la primera a la región del nordeste 
argentino y las restantes a la región pampeana. Constaba de dos cierres frontales con centrales hidroeléctricas, y uno lateral con funciones de contención: al Norte, a la altura de la ciudad de Goya (Corrientes), se ubicaba el cierre Patí; al Sur entre Romang (Santa Fe) y Villa Urquiza (Entre Ríos) se proyectaba el cierre "Chapetón". El cierre lateral recorría la margen santafesina (de cota baja y sin barranca) conformando un embalse que afectaría (según cálculos estimados) unas 700 a 760 mil hectáreas (Figura $N^{\circ} 1$ ).

El área afectada, con características de humedal, estaba constituida por el cauce principal del río Paraná y su amplia planicie aluvial, la cual incluye áreas de pantano, islas y tierras agrícolas de pequeña escala. Las poblaciones que iban a ser indirectamente afectadas (es decir, aquellas que sustentaban su economía y cultura en el área a inundar) sumaban un total de trece: siete en Santa Fe, cuatro en Entre Ríos y dos en Corrientes.

El emprendimiento hidroeléctrico se concibió originalmente como una infraestructura de relevancia regional, prometiendo la generación anual de entre 33.000 y $48.000 \mathrm{GW} /$ horas de energía (más del doble que Yacyretá). Junto a la obra principal, se planeaban construir una serie de obras

Figura $\mathrm{N}^{\circ} 1$

Plano histórico de ubicación del embalse y cierres

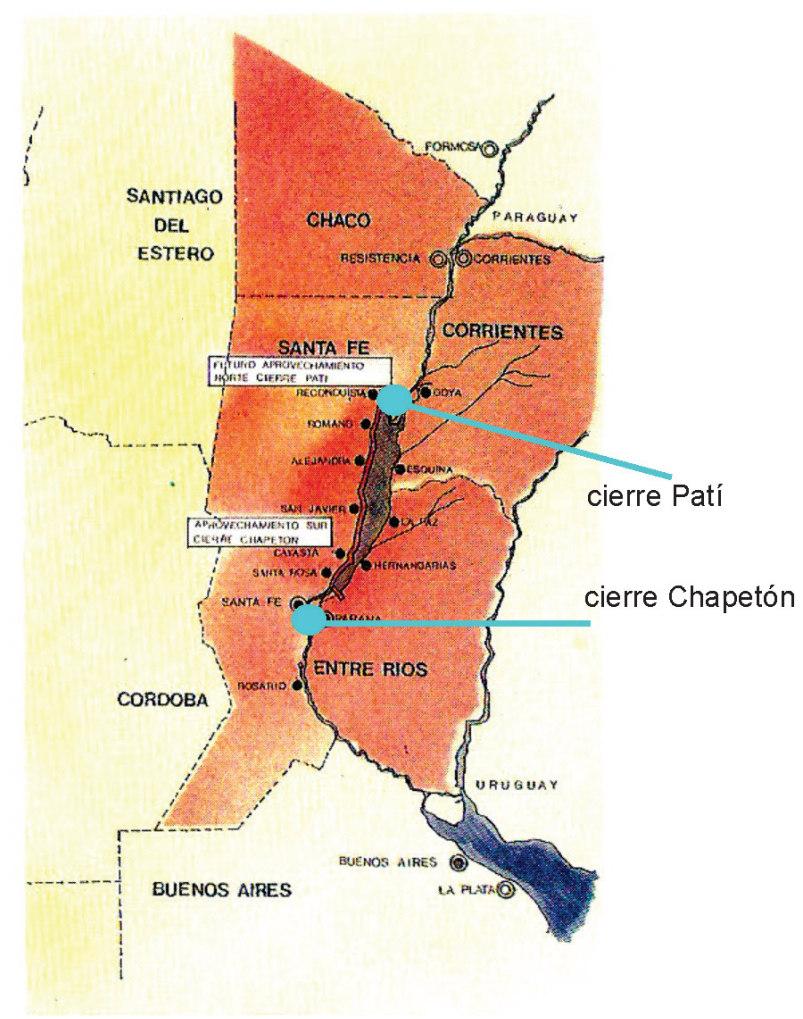

Fuente: Agua y Energía, 1980. 
complementarias con diversos fines: contención de crecidas fluviales, infraestructura ferrovial, proyectos turísticos, entre otros, siguiendo una fórmula bastante usual por ese entonces.

Algunos factores contribuyeron a que el proyecto fuese demorado: políticas energéticas que privilegiaron la producción hidroeléctrica en la región Comahue en la década de 1960 (Azcoitía y Nuñez, 2014); discontinuidades de orden político institucional (golpes de estados que se alternaron con gobiernos democráticos) junto a una serie de crisis económicas por las que atravesó Argentina en las décadas de 1960 y 1970. Recién durante la última dictadura militar (1976-1983), y en el contexto de la crisis del petróleo, el PHPM cobraría impulso (Rausch, 2016). Esto se debió, en gran parte, a "factores externos" (Brunstein, 1987:11), vinculados a la existencia de excedentes en el mercado financiero como consecuencia de las variaciones en el precio del petróleo durante esos años. A pesar de todo, el PHPM no llegó a concretarse por desavenencias posteriores del gobierno argentino con el soviético, junto a las presiones de Estados Unidos para que Argentina cortara tales relaciones (Gilbert, 1994). Con el advenimiento del gobierno democrático, en 1983, se presentaría un nuevo panorama internacional en el que se había acrecentado la dependencia económica del país (traducida en la deuda externa contraída por el gobierno dictatorial anterior) con el Fondo Monetario Internacional. La imposibilidad de financiar la obra determinó su aplazamiento, hasta que en 1986 fue desmantelada la empresa Agua y Energía, como consecuencia de las políticas de ajuste estatal implementadas por el Ministerio de Economía (Norte, 08.06.1986). Este hecho marcó la suspensión del proyecto.

\section{El Proyecto de Desarrollo Económico Paraná Medio en el programa de la Hidrovía Paraguay-Paraná: neoliberalismo y nuevas escalas territoriales durante la década de 1990}

La década de 1990 en Argentina (como así también en numerosos países de economías periféricas) estuvo marcada por diversos procesos de reestructuración económica, política y administrativa de corte neoliberal, que se tradujeron en medidas de ajuste y disciplina fiscal, en el desmantelamiento de instituciones y marcos regulatorios estatales, en la privatización de servicios (Ferrocarriles Argentinos, Agua y Energía, entre otras) y en la creación de las bases legales para facilitar la inversión extranjera. Este proceso de reforma fue prescripto por organismos internacionales (el Fondo Monetario Internacional, el Banco Mundial y el Departamento del Tesoro de Estados Unidos) en lo que se conoció como Consenso de Washington (1989).

Por otra parte, hacia 1991, se firmaba el Tratado de Asunción por el cual se creaba el MERCOSUR, consistente en un proceso de integración económica gestado inicialmente entre Brasil, Argentina, Paraguay y Uruguay. Se conformaba así, una nueva escala (supranacional) de operaciones económicas, una de cuyas manifestaciones territoriales fue el impulso al proyecto de la Hidrovía Paraná-Paraguay (HPP), considerada una gran autopista acuática de $3400 \mathrm{~km}$ a lo largo de los ríos Paraguay y Paraná (Figura $N^{\circ} 2$ ).

Según señaló Lovera Rivas (1997:3), la hidrovía fue concebida como la "médula espinal del proceso de integración regional MERCOSUR". El sistema hídrico que componen los ríos Paraguay y Paraná comunican Bolivia, Brasil, Paraguay, Uruguay y Argentina, y posee una extensión de 
3.442 km. desde Puerto Cáceres en Brasil hasta Nueva Palmira, en Uruguay. El programa general de la HPP consistía en una serie de obras de transporte vial y vías navegables, infraestructura hidroeléctrica, gasífera y refuncionalización de puertos. Este programa se orientaba a garantizar la conexión Norte-Sur entre los países y a ofrecer salida al mar a los que carecieran de la misma, contribuyendo a la expansión de la frontera agrícola-ganadera sobre áreas que hasta ese momento se encontraban bajo dominio de comunidades indígenas.

Con este programa de escala supranacional se sentaban las bases para el desarrollo del modelo extractivista-agroexportador, basado en el cultivo de soja transgénica, que se consolidaría en el siguiente siglo (Schapper, 1999; Castro Herrera, 2002).

Según consideró la Unión Internacional para la Conservación de la Naturaleza (IUCN, en inglés), la HPP tendría efectos considerables en las tierras que atravesase, con el incremento de la erosión y de los materiales de desecho (muchos de ellos contaminantes) y la destrucción irreparable de los territorios indígenas y campesinos, como resultado de la deforestación y el uso intensivo de agroquímicos. Por otro lado, la consultora Taylor-Golder-Consular-Connal (Module B2 of the HPP, 1997) señalaba que la HPP transformaría el régimen hidrológico, deteriorando la calidad del agua con pérdida del Humedal Pantanal, de la biodiversidad local, regional y global. Estaba claro que el megaproyecto beneficiaba selectivamente a grandes grupos económicos, como el de la construcción civil, el de navegación, y el agronegocio a cargo de las multinacionales Monsanto, Risco, Cargill y Syngenta; y este fue el marco económico y territorial en el que se presentaba, por segunda vez, el PHPM.

El 14 de marzo de 1995 se reunía el presidente argentino con los gobernadores de las provincias involucradas, para dar renacimiento al PHPM (EI Litoral, 21.03.1995). Un año más tarde, la prensa nacional y local informaban sobre la presentación del proyecto por parte del consorcio de empresas norteamericanas conocido como Energy Developers International (EDI) al gobierno nacional y los gobernadores (Clarín, 10.03.1996; El Litoral, 19.03.1996).

El PHPM se contemplaba dentro del "Plan Quinquenal 1995-1999", pero a diferencia de la versión anterior concebida como obra estatal, la nueva propuesta se presentaba como una inversión privada, cuyo marco legal para operar y pactar la concesión era facilitado por el propio Estado nacional. El proyecto contenía algunas modificaciones respecto al anterior, relativas al sistema constructivo (antes de hormigón in situ y ahora de módulos premoldeados de acero producidos en Estados Unidos por una de las empresas del consorcio) así como también la introducción de un sistema de 48 turbinas. Además de la obra hidroeléctrica, se proyectaban otras complementarias consistentes en infraestructura vial, puentes, refuncionalización de puertos y apertura de canales navegables a buques "Panamax", todo ello en función de los objetivos de la HPP.

Apenas presentado el proyecto, el gobierno nacional lo elevó a obra "de interés nacional" $\mathrm{y}$ creó la Comisión de Evaluación y Seguimiento del Proyecto de Desarrollo Económico Integral para la zona del Paraná Medio. La misma estaba integrada por ministros, legisladores, representantes de los gobiernos de las provincias involucradas y por la Comisión de Coordinación Interjurisdiccional del Programa HPP. El convenio del gobierno nacional con EDI preveía que este último sería 
Figura $\mathrm{N}^{\circ} 2$

El proyecto de la Hidrovía Paraguay-Paraná. Ubicación en América del Sur
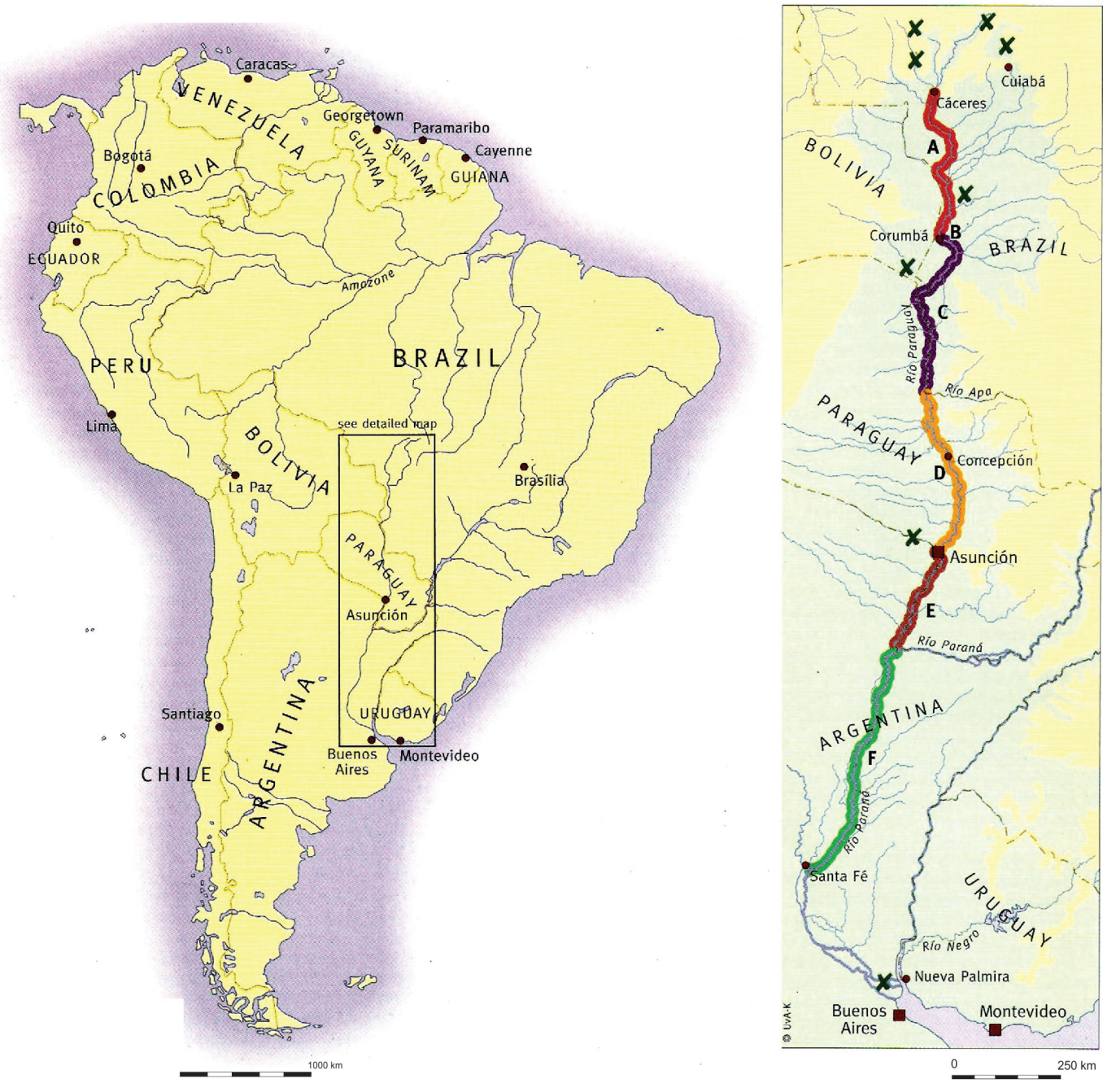

Fuente: Lovera Rivas (1997: 6-7)

usufructuario durante 50 años de las obras principales y por un lapso de 30 años de las complementarias (vías navegables, autopistas, puentes, puertos, entre otros). A cambio, el consorcio se comprometía a realizar una inversión a riesgo por un total estimado de 5 mil millones de dólares (EDI, 1996:3).

Así puede verse que el PHPM constituía una pieza de relevancia regional y subcontinental vinculada, principalmente, a la expansión del modelo de los agronegocios en Argentina. 


\section{Promesas globales, coaliciones y rechazos locales: la multiescalaridad del conflicto por el PHPM}

Brenner (1999:145), ha sugerido que, en las últimas dos décadas del siglo XX, se asistió en los países desarrollados, en vías de desarrollo y post-comunistas, al abandono, por parte del Estado, del ideal moderno de proyecto infraestructural cuyos objetivos se orientaban hacia la búsqueda de una igualdad de condiciones de vida a escala nacional. Este proceso, que se corresponde con la instauración del modelo neoliberal, fue de la mano de la atribución, a los capitales privados, de determinadas capacidades antes reservadas a los Estados nacionales (Sassen, 2010) y legitimado en gran medida por el discurso del "fracaso estatal" (Bakker, 2010: 31-35) respecto de los objetivos perseguidos durante el modelo productivista ${ }^{6}$. Así, en las dos últimas décadas del siglo $X X$, mientras los capitales comenzaban a operar a escala global y se aceleraba la circulación de dinero, de commodities y de personas, en los territorios nacionales tenía lugar un proceso de re-configuración de las escalas espaciales en que los Estados nacionales estaban organizados (Brenner, 2003). Este proceso de re-escalamiento tenía lugar, por ejemplo, mediante la incorporación de formaciones y elementos espaciales específicos (como las infraestructuras) que operarían como fijadores de los flujos globales (Harvey, 1985; Brenner, 1999). De tal modo, dichos territorios nacionales se incorporaban a la escala supranacional.

El PHPM, dentro del programa HPP, puede considerarse una infraestructura territorial que se emprendió con el objetivo de acelerar los flujos de capital e incorporar amplios territorios en una escala supranacional promovida por el MERCOSUR. Según lo expresaría EDI en el documento que acompañaba el proyecto, "en las actuales circunstancias históricas, de concreción real del MERCOSUR, esta región de la Argentina sobre la que se emplazará la obra, es estratégicamente vital para el cumplimiento de los objetivos perseguidos con el mencionado proyecto de desarrollo regional" (EDI, 1996: 6).

El conflicto puede decirse que surgió del choque entre dos coaliciones: por un lado, el capital transnacional y el Estado en sus formas de gobierno nacional y provinciales; y por el otro, las poblaciones locales (de Santa Fe y Entre Ríos), las asociaciones ambientalistas y una diversidad de instituciones civiles y educativas. La resolución a favor de estas últimas fue posibilitada, en gran medida, por dos cuestiones principales: primero, la difusión de valores e información que llevaron adelante algunas entidades ambientalistas operando a escala local, regional y global; y segundo, por la reforma constitucional de 1994, al considerar en su artículo 124 a los recursos naturales como "dominios originarios de las provincias".

A diferencia de las décadas de 1970 y 1980, en que la construcción del proyecto no presentó contrariedades a nivel social, en este nuevo intento, la noticia acerca de su realización despertó controversias e intensos debates sobre un amplio espectro de cuestiones que incluían intereses económicos, reivindicaciones nacionalistas y federalistas, luchas por el territorio considerado propio y pugnas ambientalistas.

El productivismo, según Degans (1984, citado por Marcellesi, 2008:4) puede definirse como "un sistema evolutivo y coherente que nace de la interpenetración de tres lógicas principales: la búsqueda prioritaria del crecimiento, la eficacia económica y la racionalidad instrumental que tienen efectos múltiples sobre las estructuras sociales y las vidas cotidianas". 
Una cuestión clave fue la circunscripción del conflicto en varias escalas. Existió un proceso de involucramiento, alianzas y superposición de diferentes actores sociales, económicos y políticos que operaban en diferentes escalas (local, regional, nacional y global) y que se alinearon en las coaliciones a favor y en contra de la construcción de la obra. El consorcio operaba en un nivel supranacional, cuyo marco jurídico de actuación había sido habilitado por el gobierno nacional. Este último, a su vez, negociaba la construcción del proyecto con los gobiernos provinciales, intermediando entre el consorcio y los segundos. Por otra parte, la coalición que rechazó el emprendimiento hizo uso de la Constitución Nacional, en lo referido al dominio provincial de los recursos naturales, para lograr revertir la consecución del proyecto y operó, asimismo, a varias escalas: la local representada por los habitantes de las localidades que iban a ser afectadas por el embalse; la escala regional, que incluía a las asociaciones ambientalistas congregadas en entidades mayores en Santa Fe y Entre Ríos; y la escala internacional, en la que interaccionaban algunas figuras del ambientalismo regional con otras agrupaciones afines de rechazo a los GPH (International Rivers Network) y con entidades supranacionales como la Unión Internacional para la Conservación de la Naturaleza y Naciones Unidas.

A continuación, analizaremos las construcciones discursivas de ambas coaliciones en el contexto de su producción.

\section{Energía e infraestructura para la integración económica del territorio: la coalición a favor del proyecto}

Según ha señalado Silveira (1999: 135), la década de 1990, significó para Argentina la puesta en marcha de una "nueva modernización territorial", impuesta principalmente a partir del despliegue de infraestructura que fue configurando redes de escala nacional e internacional. Fue a partir de allí, que se crearon nuevas relaciones y jerarquías territoriales que la autora considera un híbrido entre las "conexiones geográficas" anteriores y las necesidades del capitalismo mundial integrado de finales del siglo. Este proceso -sigue Silveira- fue posible en el discurrir de una "tecnoesfera" (es decir el ámbito de la técnica) y una "psicoesfera", consistente en el cuerpo de ideas, construcciones simbólicas y de sentido, que operaron como justificación y legitimación de las transformaciones territoriales.

La coalición que defendió la realización del PHPM estaba conformada por una serie de instituciones y organismos que iban a ser beneficiados política y económicamente por la obra. Además del gobierno nacional, pueden contarse a los gobernadores de las provincias Santa Fe, Entre Ríos (en un primer momento) y Corrientes, a la Bolsa de Comercio de Santa Fe y Entre Ríos, a la Comisión Santafesina de Apoyo a las Obras del Paraná Medio, al Colegio de ingenieros y a la Universidad Tecnológica Nacional.

Dos núcleos de ideas conformaban el marco de justificación para construir el emprendimiento: 1) el "salto" tecnológico y el manejo eficiente de los recursos conducirían al desarrollo de la región; y 2) la obra permitiría alcanzar la seguridad energética y de esta manera garantizaría la productividad y el desarrollo. 
La asociación entre represas y desarrollo tiene larga data y deriva de la visión utilitarista de la naturaleza que, desde la visión baconiana en adelante, "debe" ser dominada tecnológicamente. En particular, las grandes represas adquirieron, durante el siglo XX, gran significación promocionadas por los gobiernos como piezas tecnológicas al servicio del desarrollo. En la década de 1990, esta idea de organización tecnológica de la naturaleza asociada al desarrollo (ya no nacional, sino regional) tendrá algunas variaciones en función del contexto del capitalismo mundial integrado, y para el caso de Argentina, en relación tanto a su lugar económicamente periférico, como a su rol de país exportador de materias primas. El "salto" tecnológico, traducido en infraestructura, se promocionaba como "necesario" para alcanzar un supuesto desarrollo, y colocaba el énfasis en la eficiencia del manejo de los recursos (económicos, principalmente). Ya que la rentabilidad sería el parámetro para hacer viables las economías regionales, bajo el concepto de "fracaso estatal", los sectores privados y los capitales multinacionales asumirían como los principales impulsores de los grandes proyectos de infraestructura.

En este sentido, Silveira (1999:332) ha sugerido que a través de un conjunto de símbolos, ideas fuerza e imágenes, se ha construido un "discurso geográfico" ${ }^{8}$ cuyo objetivo ha sido dar cuenta de la "inevitabilidad" del modelo de modernización territorial, basado en el despliegue de los sistemas tecnológicos. Este modelo, advierte la autora, hizo uso de la persuasión y de la manipulación, creando asociaciones entre el establecimiento de una racionalidad tecnocientífica y la idea de progreso, de desarrollo regional o nacional, y la creación de empleos (Silveira, 1999: 331). Pero, además, se sustentó en la disociación entre los sistemas de objetos y los sistemas de acciones (Silveira, 1999: 332), con lo cual los objetos presentados de manera aislada han impedido observar las racionalidades por fuera de la región o el país de las cuales forman parte. A este respecto, considera Silveira, las represas han constituido un caso paradigmático.

En efecto, para el caso del PHPM, sus promotores justificaban la construcción a través de una serie de beneficios falaces: 1) aumento de puestos de trabajo en la región durante el período de construcción. En las promesas de los representantes gubernamentales, la obra significaba la creación de diez mil puestos de trabajo $(E D I, 1996)$ en un momento en que la población desocupada en Argentina llegaba al 18"\% (INDEC, 1995). Sin embargo, el sistema constructivo aportado por EDI consistía en piezas premoldeadas en Estados Unidos que requerían escasa mano de obra y tiempo de montaje; 2) generación de energía "limpia y renovable" a la región. La condición de "limpia" hacía referencia a que la represa no provocaría emanaciones de dióxido de carbono a la atmósfera, aunque ninguna de las características aplica a los GPH en ríos de llanura (como el proyecto en cuestión) debido a los sedimentos que arrastra el río y la materia orgánica que se acumula en los embalses de grandes dimensiones; 3 ) control de inundaciones y "seguridad" hídrica, un nuevo valor asociado a la eficiencia que se traducía en la calidad ambiental que supuestamente posibilitaría la represa a través del control del régimen fluvial. En realidad, se trataba de una seguridad ficticia, en tanto el valle de inundación en su condición natural funciona como regulador de las crecidas, y en cambio, su terraplenamiento colocaba a las poblaciones aledañas en serio riesgo ante cualquier desperfecto de la infraestructura en períodos de precipitaciones mayores a las usuales; 4) "recuperar" tierras para la producción agrícola. Otra falacia, siendo que en el área a inundar por el embalse ya existía producción agrícola-ganadera no intensiva, de escala familiar y adaptada a dicho régimen, que iba a ser destruida. Por lo tanto, la "recuperación" promovida en 
realidad consistía en una redistribución de beneficios desde los actores locales a los productores de mayor escala.

Es interesante observar cómo el discurso de la coalición a favor del proyecto, insistía en el beneficio de la regulación de las inundaciones, lo cual, para las localidades ribereñas (sobre todo de Santa Fe) constituía un factor ciertamente sensible.

Por otra parte, Ortiz (2008:8) ha señalado que EDI utilizó un discurso "políticamente correcto" que proponía contemplar las cuestiones ambientales, además de las económicas y técnicas, pero paralelamente sugería la realización de un estudio de impacto ecológico a cargo de una de las empresas miembros del mismo consorcio, sin posibilidad de consulta externa o consulta popular. La vinculación entre las consultoras que realizan los estudios de impacto ambiental y las constructoras de represas no es inusual, como ha advertido Mc Cully (2001:67), pero incluso no existiendo vinculación, por tratarse de consultoras privadas, en general -sostiene el autor- éstas suelen enfatizar en sus informes los aspectos favorables de las obras en función de obtener futuros contratos. A lo largo del documento de más de 30 páginas confeccionado por EDI, sólo aparece un apartado de dos páginas referido a las cuestiones ambientales, y éstas se limitan a subrayar que la energía hidroeléctrica es una fuente renovable. Es notorio el interés puesto en la sostenibilidad económica del recurso (el río), apoyándose en una versión economicista del concepto de sostenibilidad ${ }^{9}, \sin$ mayores preocupaciones por las poblaciones locales que iban a ser afectadas.

La segunda idea a extraer daba cuenta de un pasaje de la noción de "abundancia" (de recursos) que había nutrido el optimismo económico-productivista de las burocracias estatales (Kaika, 2006b:152), a una idea de "escasez" (de recursos naturales, de energía), a partir de la cual se nutrió el discurso neoliberal.

Lyla Mehta (2011:13-30) sostiene que las discusiones en materia de políticas energéticas se han mantenido siempre entre los dos pilares principales de la economía neoclásica: el miedo a la escasez y las promesas de abundancia. Mientras que en el primero se asume que las necesidades de energía son ilimitadas, aunque los medios para obtenerla son limitados (de ahí el énfasis en la eficiencia); en el segundo se considera que los mercados, la innovación tecnológica y los procesos sustitutivos resolverán dicha escasez, sin tener en cuenta el límite de los recursos.

Por su parte, Hildyard, Lohmann y Sexton (2014:40) han señalado que "cuando se naturaliza la escasez -al hacerla parte de la condición humana- se dejan de lado preguntas incómodas, por ejemplo: ¿cómo se ha creado y aún se crea deliberadamente la demanda hacia fuentes específicas de energía?". Para los autores, es la escasez la que debería ser explicada, sin embargo, bajo la lógica capitalista se ha convertido en la justificación para la creación de mercados energéticos. De este modo, la idea de creciente demanda (de bienes, de energía, de recursos) ha instalado, desde finales del siglo XX (principalmente a partir de la crisis del petróleo), la noción de escasez que se ha difundido con mayor intensidad en los últimos años, y según sostiene Kaika (2006b), ha

El desarrollo "sostenible" fue definido en el Informe Brundtland (1987) como aquel que ofrece: 1) garantía para las generaciones futuras de un mundo físico-material y de seres vivos igual o mejor que el actual; y 2) equidad social para las generaciones presentes. Bajo el neoliberalismo, las políticas ambientales han sido orientadas por una visión eminentemente técnica que pone el énfasis en la búsqueda de cuidar las características físico materiales relegando los aspectos sociales (Foladori, 1999). 
justificado el eficientismo empresarial, alentando la racionalización y la privatización en el manejo de los recursos naturales.

Para el caso de caso del PHPM, el consorcio justificaba la obra en un (supuesto) futuro desarrollo económico del país, cuya condición era el incremento en la generación de energía, tanto para la producción interna como para su exportación a países limítrofes: “Un país como Argentina necesita energía para desarrollarse al máximo. Un país como Argentina con energía en abundancia a un costo fijo y razonable, se convertiría en generador y exportador de energía" (EDI, 1996: 23). Dicho de otro modo: a partir de una idea de abundancia futura (y, por lo tanto, de escasez presente), se creaba una demanda para justificar la construcción de la obra hidroeléctrica en términos de beneficios socioeconómicos - "una ecuación donde todos ganan", expresaría EDI (1996). Aunque dichos beneficios, como se observó antes, estaban destinados a determinados sectores productivos y, sobre todo, al consorcio durante el tiempo de la concesión.

\section{"Un río que nació para correr no lo deben detener"10: la coalición opo- sitora en Santa Fe y Entre Ríos, y el surgimiento de la contestación socio-ambientalista}

En 1995, el presidente argentino Carlos Menem (1989-1999) se reunió con los gobernadores de las provincias de Santa Fe (Jorge Obeid), Entre Ríos (Jorge Busti) y Corrientes (Romero Feris) así como también con los representantes de EDI, Nicholas Brady y George Bush (hijo). El objetivo era dar curso al PHPM (El Litoral, 21.03.95). Sin embargo, las acciones de rechazo a la implementación de la obra comenzarían a visibilizarse un año después, junto a la presentación formal de la propuesta por parte del consorcio (El Litoral, 19.03.96). Mientras el diario Clarín emitía la noticia exponiendo las características principales del proyecto y calificándola como una obra para el "desarrollo de la región" (Clarín, 16.03.96); el diario Página 12 titulaba un artículo: "Como si no nos bastara con una Yacyretá, Menem quiere otra más" (Página 12, 20.03.96). En Santa Fe, el vespertino El Litoral tomaba algunas precauciones sobre su posicionamiento: "Paraná Medio: otra vez y con las mismas dudas". Aquí, el columnista declaraba:

"la identidad básica del proyecto reactiva viejas dudas ecológicas que, en principio, se consideraban superadas. La desaparición de cientos de islas y de una extensa franja de campos costeros a causa del gigantesco lago que habría de formarse y de la presa lateral que lo contendría en la orilla baja santafesina, abre numerosos interrogantes"; y expresaba su desconfianza: "pero cuidado con los cantos de sirenas, que las necesidades y urgencias de hoy no nos tapen el bosque de indeseadas consecuencias futuras" (El Litoral, 21.03.96).

En esos días, en la ciudad de Paraná, se reunía un conjunto bastante heterogéneo de militantes ambientalistas y miembros de ONGs para organizar un frente opositor al emprendimiento, creándose la Asociación de Entidades Ambientalistas (AEA). A ella se sumarian otras agrupaciones, también ambientalistas, de Santa Fe y Entre Ríos, dando curso a las acciones opositoras. Coincidimos con Arach (1999:45) cuando destaca el carácter confuso y caótico de la coalición entrerriana, sometida a tensiones de diverso origen. Un cariz semejante adquiriría las movilizaciones en Santa Fe, no pudiendo identificarse un "curso" de acciones organizadas, sino más bien una "galaxia" de

- Fragmento de obra musical compuesta por el cantautor santafesino Orlando Vera Cruz, apropósito del proyecto Paraná Medio. 
actividades, que coincidían en rechazar la represa, pero que, en ocasiones, se contradecían en el accionar o simplemente actuaban de manera autónoma (cada asociación por sí misma).

Dentro de esta multiplicidad opositora, pueden identificarse tres instituciones cuya participación fue visible en mayor grado: en Santa Fe la Fundación PROTEGER y el Ce.Pro.Nat (Centro de Protección a la Naturaleza); y en Entre Ríos la antes mencionada AEA. A la actividad de estas organizaciones se sumaron también diversos actores sociales, como funcionarios públicos, religiosos y del ámbito educativo, por mencionar los más relevantes. La heterogeneidad social que conformaba la coalición opositora se traducía en una diversidad de ideas y motivos por los cuales se oponían. En las construcciones discursivas de la misma, pueden identificarse una serie de enfoques que en ocasiones de mixturaban: 1) socio-ambientalista, centrado en la defensa de la calidad ambiental, en vinculación a la biodiversidad del área y a la cultura ribereña (costumbres, usos del ambiente); 2) conservacionista, de tendencia biologicista, preocupado principalmente por la pérdida de la fauna y la flora del área así como del paisaje fluvial; 3) nacionalista-productivista, cuyo cuestionamiento no se dirigía a la represa sino al carácter "extranjerizante" y desventajoso de la concesión; 4) sanitario-higienista, centrado en las enfermedades que produciría el anegamiento de las 700 mil hectáreas"1; y 5) federalista, cuyo reclamo principal era el "avasallamiento" de los derechos provinciales sobre los recursos naturales llevado a cabo por el Poder Ejecutivo Nacional. Esta posición se dio principalmente en Entre Ríos y retomaba la vieja antinomia unitario/ federal constitutiva de la organización política argentina.

Sin embargo, fueron las ONGs ambientalistas quienes canalizaron los reclamos y así, el discurso opositor se mantuvo en los carriles de su ideario. Sus reivindicaciones podrían sintetizarse en tres ideas: 1) la protección del río en tanto recurso fundamental para la permanencia de las formas de vida locales (basadas principalmente en la pesca y la ganadería de isla); 2) la protección de los valores paisajísticos del ambiente fluvial; y 3) la preservación de la soberanía sobre el río, en vistas del convenio favorable al consorcio norteamericano.

En líneas generales, estas ideas pueden considerarse, por un lado, afines a la llamada "modernización ecológica"12, con claras influencias de los postulados del informe Brundtland (1987), y por el otro, influenciadas por los movimientos de justicia ambiental (principalmente del brasileño "Movimento dos Atingidos por Barragem"). En este sentido, los discursos se construyeron en torno a la preocupación por preservar los modos de vida de las localidades que serían afectadas y, por lo tanto, su economía de pequeña escala y el río en tanto valor que nuclea y organiza a la comunidad. De ahí que Adrián Linari, escritor perteneciente a una de las localidades santafesinas que iban a ser afectadas por el embalse, señalara que el río "para una cultura seminal, es parte necesaria del propio ser" y que el proyecto hidroeléctrico alteraría "de un modo violento" el medio en que tiene lugar la cultura del "islero"13 (El Litoral, 21.12.96). Pero, asimismo, la coalición enfatizaba en la necesidad de resguardar el mundo físico-material y los seres vivos para las generaciones futuras, a través del aprovechamiento sostenible del río y sus recursos a cargo de las poblaciones locales.

\footnotetext{
Como Esquistosomiasis, Paludismo y Denge

12 La modernización ecológica puede concebirse como un proceso de apropiación utilitarista de los valores ecologistas para su implementación en las políticas públicas. La misma considera que el crecimiento económico y la resolución de problemas ambientales pueden conciliarse en los principios de un capitalismo sostenible (Hajer, 1995:26).

13 Así son denominados localmente a quienes viven en el área ribereña del Paraná
} 
La lucha anti-represa asumiría formas variadas en las provincias de Santa Fe y Entre Ríos: marchas de pobladores en las localidades ribereñas, cartas a los gobernadores, recolección de firmas, reuniones de información y debate público, difusión de la problemática a través de visitas instituciones educativas, en folletos o en la prensa de Paraná y Santa Fe. Cabe destacar que la prensa nacional apenas se hizo eco de la actividad contra el proyecto hidroléctrico, y según expresaron algunos participantes del movimiento opositor, en los diarios de Buenos Aires "no los recibieron" (entrevista a Jorge Cappato, 2013).

El primer volante confeccionado por la AEA, expresaba: "Los ríos son especies en extinción: No a la represa contra el Paraná Medio. Sí a la vida". Arach (1999: 43) advierte en este lema algunos elementos que considera básicos de la lucha ambientalista de esos años: en primer lugar, destaca la referencia al río como una entidad viva cuyo represamiento significaría su muerte (extinción). Esta consideración se repetía frecuentemente, asociando "agua estancada" (el embalse) con "muerte"; o al tramo medio del río Paraná con "riñón del Paraná entero" (Halle, 1996:9). En segundo lugar, señala la redefinición del término "paraná medio", que hasta ese momento se asociaba al proyecto hidroeléctrico y no al río. Y, en tercer lugar, reconoce la instauración de un sentido de la lucha contra la represa como una afirmación de vida, contraponiéndose a la construcción del embalse.

Un acontecimiento mediático que ha permanecido en la memoria de sus participantes, fue la travesía de 22 días de duración que dos pescadores realizaron en 1996, uniendo la represa Yacyretá (Corrientes) con la capital entrerriana. También el cantautor santafesino Orlando Vera Cruz se manifestó, escribiendo una polca llamada "Paraná Entero" (1997). En sus estrofas se expresa la preocupación por los efectos de la obra en el cambio de la dinámica fluvial:

"Un río que nació para correr/no lo deben detener/Si se afina en una senda/si la montaña lo apresa/puede hacerse una represa/Pero donde el llano talla/no debe hacerse muralla...", haciendo referencia a que el proyecto en cuestión no se encontraba en sitio de represa ${ }^{14}$ ) y a sus catastróficos efectos en el ambiente: "...los árboles y nidos/se mueren sumergidos/ las aves sobrevuelan/un lago sin sentido/espejo artificial/del hombre irracional."

Este último verso es particularmente interesante, ya que en el mismo se subvierte la antinomia moderna: técnica-racional/naturaleza-irracional, colocando del lado de la racionalidad, la preservación del río.

Siguiendo una fórmula ya utilizada por los movimientos ambientalistas de Brasil, la coalición confeccionó, además, algunas "declaraciones locales" en las que se difundieron los motivos por los cuales la población rechazaba la obra (Declaración de Santa Fe, 1996, Declaración de Helvecia, 1996, Declaración de Cayastá, 1996).

Dos eventos internacionales fueron clave: para legitimar el reclamo fue importante la Cumbre de la Tierra (Río de Janeiro, 1992), y para visibilizarlo a nivel internacional, el I Encontro Internacional dos Povos Atingidos por Barragems, organizado por el MAB (Curitiba, 1997). Mediante la parti-

El sitio de represa es un concepto técnico que se define cuando el río se encuentra limitado por dos paredes naturales, como, por ejemplo, en zonas montañosas. 
cipación de algunos integrantes de la coalición opositora en ambos, se incorporaron a la lucha conocimientos sobre experiencias semejantes en otros países (Estados Unidos y Brasil, por ejemplo) así como también instrumentos para encauzar y fortalecerla (entrevista a Jorge Cappato, 2013).

Hacia junio de 1996, las acciones opositoras que hasta ese momento habían corrido por cursos informales y de manera bastante caótica, comenzaron a canalizarse por las vías institucionales y judiciales. La AEA realizó primero una presentación en la Defensoría del Pueblo de Paraná, y en febrero de 1997, un grupo de abogados ambientalistas elevaron un recurso de amparo ante la Justicia Federal de Paraná para declarar anticonstitucional el Decreto Nacional que consideraba "de interés público" la realización del proyecto. Al obtenerse, en mayo de 1997, el fallo a favor (entrevista a Jorge Daneri, 2015), la coalición prosiguió a dar curso a un proyecto de ley provincial que prohibía la construcción del proyecto en el tramo del río Paraná de dominio entrerriano ${ }^{15}$. El 25 de setiembre de 1997 se sancionaba la Ley provincial n 9092, por la cual se declaraba a la provincia de Entre Ríos "libre de nuevas obras de represamiento sobre los ríos Paraná y Uruguay concordantes con las facultades dispuestas en los artículos $1^{\circ}, 5^{\circ}, 41^{\circ}$ y $124^{\circ}$ de la Constitución Nacional". Con esta ley se suspendía el proyecto Paraná Medio en la medida que se prohibía la construcción del cierre Sur en el dominio entrerriano.

Ahora bien, más allá de las acciones de la AEA en Entre Ríos y de las organizaciones ambientalistas en Santa Fe, que fueron claves para la resolución del proceso favorable a sus demandas, los sucesos deben dimensionarse a la luz del contexto histórico trazado por una serie de cuestiones de orden internacional y nacional:

1) Hacia las dos últimas décadas del siglo $X X$ a nivel mundial, los efectos nocivos de las grandes represas en los ámbitos locales comenzaron a ser difundidos con mayor intensidad, a la par de la conformación de movimientos sociales anti-represa (Mc Cully, 2001:339373). Asimismo, durante la década de 1990, el pensamiento ecologista (en sus diferentes versiones) tuvo gran difusión en América Latina, principalmente a partir del auge de las ONGs, que en los países "del Sur" se articularon, en numerosas ocasiones, con los movimientos sociales "de base" (Reboratti, 2000). Este contexto de mayor difusión posibilitó la discusión sobre los emprendimientos, pero también sobre problemáticas más generales (como el desarrollo, el productivismo, la organización tecnológica de la naturaleza), las cuales hasta hacía poco tiempo eran indiscutidas y constituían el eje argumental de las políticas energéticas llevadas adelante por los gobiernos.

2) Durante la década de 1990, la Comisión Mundial de Represas (WCD, en inglés), financiada por el Banco Mundial, llevó a cabo estudios en diferentes países sobre deterioro provocado en los ámbitos de implantación de grandes represas. Los resultados fueron difundidos en el informe publicado en el año 2000, luego de algunas controversias con movimientos ambientalistas locales que bregaban por tener participación en el mismo (Mc Cully, 2001). En paralelo, el Banco Mundial, principal organismo financiador de estos emprendimientos en los países en vías de desarrollo, ya no ofrecía préstamos para estos fines.

El rumbo detallado que fueron tomando las acciones de la AEA hasta la sanción de la ley puede verse en Arach (1999). 
3) En 1994 se reformaba la Constitución Nacional Argentina. La nueva versión contemplaba un rol más activo de la población en distintas instancias legales y judiciales (Art. 39 y 43). Además, se les daba, a las provincias, el dominio originario de los recursos naturales (Art. 124). Esta reforma, como se vio antes, permitió llevar adelante el reclamo judicial contra la construcción del proyecto hidroeléctrico en Entre Ríos, y luego la confección del proyecto de ley provincial.

5) La sanción de la ley No 9092 fue posible, en parte, por el apoyo del gobernador entrerriano, Jorge Busti ${ }^{16}$, que marcó la diferencia con el gobernador de Santa Fe, especialmente interesado por la realización de la obra.

\section{Ponderaciones finales del conflicto por el Paraná Medio en los umbrales del modelo neoextractivista}

Las grandes represas se promocionan como infraestructuras fundamentales para la generación de energía, el abastecimiento de agua para la agricultura, la industria y la urbanización, el control de las inundaciones y el favorecimiento de la navegación. Incluso, se han promovido (como es el caso del PHPM) como obras impulsoras de actividades recreativas y turísticas. Sin embargo, tanto la experiencia de las poblaciones afectadas como los estudios difundidos por diferentes organizaciones e investigadores, han demostrado que tales "bondades" tienen altísimos costos en términos ambientales y sociales, e incluso políticos y económicos cuando las obras se llevan adelante en medio de hechos de corrupción, como son los ejemplos de Yacyretá (Lins Ribeiro, 1994) y Tres Gargantas (Mc Cully, 2001).

Mc Cully (2001:36) ha identificado los principales impactos de las grandes represas que, en líneas generales, pueden sintetizarse en la fragmentación, tanto del ecosistema fluvial (aguas arriba y debajo del embalse), como de las formas sociales existentes, asociadas al mismo. Pero, además, el autor advierte que las grandes represas pueden considerarse "un experimento enorme, a largo plazo y mayormente irreversible y sin control" (Mc Cully, 2001:37), debido a las incertidumbres sobre los impactos de la ingeniería hidráulica y a los efectos que se visibilizan, en numerosas ocasiones, en el largo plazo.

A dichos efectos, se le suman los problemas del sistema de ingeniería y el mal funcionamiento del mismo, en general derivados de estudios previos insuficientes que no contemplan las características singulares del río y el lugar de implantación. Así, por ejemplo, suele suceder que la capacidad de generación energética resulta sensiblemente menor a la estimada por acumulación de sedimentos, se producen desmoronamientos por desconocimiento de las características geológicas de las paredes naturales, e incluso movimientos en la tierra que a su vez repercuten en fisuras en la misma represa. De ahí que, desde la década de 1980, se han vuelto visibles los numerosos conflictos socio-territoriales, inherentes a la construcción de estas megaobras, creándose diversas organizaciones "anti-represa" en todo el mundo.

En opinión de participantes del conflicto, dicho viraje se vinculó a las elecciones legislativas que tendrían lugar en octubre de 1997, y en las cuales el gobernador esperaba resolución satisfactoria para su partido (entrevistas a Jorge Capatto, 2013 y Jorge Daneri, 2015). 
Para el caso del PHPM, según las asociaciones ambientalistas participantes en el conflicto, la construcción del embalse traería graves consecuencias ambientales que redundarían en el desarraigo de miles de personas (calculadas en más de 30 mil) cuya fuente de subsistencia y trabajo es el río y la planicie aluvial (Declaración de Santa Fe, 1997). Sin embargo, era alto el grado de incertidumbre sobre estas consecuencias, ya que no se habían realizado estudios profundos de ecología. Sólo se contaba con un Informe de Ramón Margalef (1981) quien remarcaba la imprevisibilidad del proyecto. El "gigante dormido", como le llamaban al río Paraná los promotores de la represa en la década de 1970, era en realidad, hacia la década de 1990, un "gigante desconocido" (Jorge Cappato, s/f).

Asimismo, el PHPM en esta última década, debe leerse a la luz del proceso de emergencia de la escala transnacional que, en las dos últimas décadas del siglo XX, implicó dos procesos concomitantes: por un lado, la concentración de los capitales en los centros de comando (generalmente las ciudades globales), y por el otro, la descentralización de las operaciones productivas. Este último proceso se tradujo espacialmente en la creación de zonas de inversión y extracción (de recursos naturales) en países con economías débiles, que poseían recursos naturales (y se encontraban ávidos de recibir inversiones), y se dio a la par del despliegue de una base tecnológica funcional a dichos fines.

Puede decirse que el conflicto en torno a la construcción del PHPM se ubica en los umbrales del modelo neoextractivista del siglo XXI. La década de 1990 puede considerarse el momento en que se sentaron las bases para el despliegue de este modelo (por ejemplo, a través de la creación de marcos normativos y jurídicos, y de la confección de proyectos de infraestructura de escala transnacional) que sería profundizado bajo otras formas, por un gobierno de corte progresista, en el siguiente siglo (Gudynas, 2009). Pero en el contexto del neoliberalismo, cuya visión economicista y eficientista del territorio avanzaba sin consideraciones hacia las economías locales y regionales, la propuesta del emprendimeinto hidroeléctrico tuvo su correlato en el amplio rechazo social. Esta movilización puede considerarse en la transición entre las formas más tradicionales del activismo ambientalista y las más innovadoras, que Svampa (2010 y 2011) ubica en el siglo XXI, formando parte de lo que denominó el "giro ecoterritorial" de las luchas.

Lo interesante de este momento fue que, aquello que en un principio apareció simplemente como un rechazo a la obra hidroléctrica, dio lugar a un sinnúmero de debates y cuestionamientos en torno al modelo de desarrollo vigente. Este proceso, que según ha señalado Antonelli (2009), va de la conformación de "comunidades del no" a la radicalización y ampliación de la plataforma representativa y discursiva hacia el cuestionamiento del modelo de desarrollo hegemónico basado en la mercantilización de la naturaleza (que Svampa y Viale han identificado en los movimientos anti-minería del siglo XXI), pudo observarse incipientemente en el caso de la lucha anti-represa en cuestión. Aquí, entre la caótica producción de la coalición opositora, aparecían escritos y artículos en la prensa que planteaban la necesidad de observar críticamente los valores hegemónicos y la noción de desarrollo asociado a la mercantilización de la naturaleza.

Otra cuestión sobresaliente de la lucha por el Paraná Medio (y que la diferencia de otras luchas socio-ambientalistas posteriores en Argentina que han fracasado) fue la resolución del conflicto a favor de la contestación social. Dicho triunfo, se dio en un contexto mundial de difusión de los valores ambientalistas y cuestionamiento a los distintos modelos desarrollistas de las décadas precedentes. A su vez, el discurso neoliberal que atravesaba tanto las esferas gubernamentales 
como la propuesta de EDI, haciendo énfasis en la intervención privada del curso fluvial por parte de capitales de escala multinacional, junto al acotado papel del Estado que dejaba en manos de EDI, tanto la consecución del proyecto como la totalidad de los beneficios económicos, se encontró con una oposición irreductible, conformada por amplios sectores sociales que se alinearon al rechazo de la represa más allá del interés por preservar el ambiente (los sindicatos, por ejemplo, se oponían a la participación del capital foráneo en el emprendimiento).

Puede verse así, que la fuerza del discurso anti-represa en el caso en cuestión se debió, en gran parte, a su misma conformación heterogénea, que integraba una variedad de reivindicaciones, a las cuales se fueron adhiriendo amplios sectores sociales, aunque no compartiesen los ideales del ambientalismo tradicional. Pero, asimismo, se debió al re-escalamiento que se produjo en el modus operandi de un sector de las ONGs ambientalistas, que comenzaron a integrar redes trascendiendo la escala local, a la que, hasta ese momento, habían limitado sus luchas.

\section{Referencias bibliográficas}

AIDA. Grandes represas en América ¿peor el remedio que la enfermedad? Principales consecuencias ambientales y en los derechos humanos y posibles alternativas. México: AIDA/CS Fund, 2009.

AGUA Y ENERGÍA. Proyecto Integral Paraná Medio. Santa Fe, 1980.

ANTONELLI, M. Minería transnacional y dispositivos de intervención en la cultura. La gestión del paradigma hegemónico de la "minería responsable y desarrollo sustentable". En: SVAMPA, M. y ANTONELLI, M. (editores). Minería transnacional, narrativas del desarrollo y resistencias sociales. Buenos Aires: Biblos, 2009, p. 51-101.

ARACH, O. La lucha sobre el río: un análisis de la oposición a la represa del Paraná Medio (Entre Ríos 1996-1997). Posadas: Tesis Maestría en Antropología social, Universidad Nacional de Misiones, 1999.

AZCOITÍA, A. y NUÑEZ, P. Las represas hidroeléctricas de la región Comahue: expectativas de un desarrollo parcial. Agua y Territorio, 2014, N 4, p. 12-22.

BAKKER, K. Privaticing Water. Governance Failure and the World's Urban Water Crisis. Ithaca and London: Cornell University Press, 2010.

BRENNER, N. Globalisation and reterritorialisation: the re-scaling of urban governance in the European Union. Urban Studies, 1999, Vol. 36, Nº 3, p. 431-451.

BRENNER, N. La formación de la ciudad global y el re-escalamiento del Estado en la Europa occidental post-fordista. Eure, 2003, Vol. 29, No 86, p. 5-35.

BRUNSTEIN, F. Las grandes inversiones públicas y el problema del desarrollo regional. En: ROFMAN, A. y Brunstein, F. Los grandes proyectos y el espacio regional. Presas hidroeléctricas y el sistema decisional. Buenos Aires: Cuadernos del CEUR, N 19, 1987, p. 1-41. 
CASTRO HERRERA, G. Naturaleza, sociedad e historia en América Latina. En: ALIMONDA, H. (compilador). Ecología Política. Naturaleza, sociedad y utopía. Buenos Aires: CLACSO, 2002, p. 83-99.

CATULLO, M.R. Poder, participación y niveles de decisión en proyectos de gran escala. En: $V$ Congreso de Antropologia Social, La Plata, julio-agosto 1997. Disponible en Internet: http://www.equiponaya.com.ar/congresos/contenido/laplata/LP3/28.htm

CATULLO, M. R. Estudios de los efectos sociales de la construcción de represas hidroeléctricas y la conformación de redes científicas en América Latina (1992-2004). Revista ILHA, 2008, Vol. 10, No 1, p. 273-292.

CATULLO, M.R. y COUN, E. Estudios de impactos sociales en el Mercosur. Procesos relocalizatorios, nuevos espacios urbanos y reconstrucción de redes de relaciones sociales. Cuadernos de Antropología Social, 2002, N 15, p. 49-69.

EDI. Proyecto de Desarrollo Económico Integral del Paraná Medio con beneficio directo para las provincias de Santa Fe, Entre Ríos y Corrientes. Luisiana, 1996.

FERNÁNDEZ, M. y CARRILLO, M. (coordinadores). América sumergida: impactos de los nuevos proyectos hidroeléctricos en Latinoamérica y el Caribe. Barcelona: Icaria, 2010.

FERNÁNDEZ, M. Energía hidroeléctrica y sostenibilidad. FERNÁNDEZ, M. y CARRILLO, M. (coordinadores). América sumergida: impactos de los nuevos proyectos hidroeléctricos en Latinoamérica y el Caribe. Barcelona: Icaria, 2010, p. 13-36.

FOLADORI, G. Sustentabilidad ambiental y contradicciones sociales. Ambiente \& Sociedade, 1999, No 5, p. 19-34.

GILBERT, I. El oro de Moscú. Historia secreta de la diplomacia, el comercio y la Inteligencia soviética en la Argentina. Buenos Aires: Planeta, 1994.

GRUPO PERMANENTE DE TRABAJO SOBRE ALTERNATIVAS AL DESARROLLO. Más allá del DesarroIlo ( $2^{\circ}$ edición). Buenos Aires: Fundación Rosa Luxemburgo/América Libre/Abya Yala, 2012.

GUDYNAS, E. Diez tesis urgentes sobre el nuevo extractivismo. Contextos y demandas bajo el progresismo sudamericano actual. Contextos y demandas bajo el progresismo sudamericano actual. En: CENTRO ANDINO DE ACCIÓN POPULAR Y CENTRO LATINOAMERICANO DE ECOLOGIA SOCIAL. Extractivismo, política y sociedad. Quito: CAAP-CLAES, 2009, p. 187-225.

GUDYNAS, E. Derechos de la naturaleza. Ética biocéntrica y políticas ambientales. Buenos Aires: Tinta Limón, 2015.

HAJER, M. The Politics of Environmental Discourse. Ecological Modernization and the Policy Process. Oxford: Oxford University Press, 1995.

HALLE, O. Contrapunto sobre la represa del Paraná Medio. La tierra sin mal. Santa Fe, 1996. 
HARVEY, D. The geopolitics of capitalism. In: GREGORY, D. \& URRY, J. (editors). Social relations and spatial structures. London: Macmillan, 1985; p. 128-163.

HILDYARD, N.; LOHMANN, L. y SEXTON, S. Seguridad energética ¿para qué? ¿para quién? Madrid: Libros en Acción, 2014.

KAIKA, M. City of Flows: Modernity, Nature and the City. London and New York: Routledge, 2005.

KAIKA, M. Dams as Symbols of Modernization: The Urbanization of Nature between Geographical Imagination and Materiality. Annals of the Association of American Geographers, 2006a, p. 276-301.

KAIKA, M. The political ecology of water scarcity: the 1989-1991. Athenian drought. In: HEYNEN, N.; KAIKA, M. \& SWYNGEDOUW, E. In the Nature of Cities. Urban Political Ecology and the Politics of Urban Metabolism. London and New York: Routledge, 2006b, p. 150-164.

KAIKA, M. Landscapes of energy: Hydropower from Techno-Natures to Retro-Natures. Harvard New Geographies, 2009, № 2, p. 103-110.

LINS RIBEIRO, G. Transnational capitalism and hidropolitics in Argentina. The Yacyretá High Dam. Florida: University Press of Florida, 1994.

LOVERA RIVAS, M. The Hidrovia: reinventing the wheel. Netherlands: Macula/IUCN, 1997.

MARCELLESI, F. Ecología política: génesis, teoría y praxis de la ideología verde. Cuadernos Bakeaz, 2008, $\mathrm{N}^{\circ}$ 85. Disponible en Internet:

https://florentmarcellesi.wordpress.com/2013/02/07/la-ecologia-politica-una-ideologia-global-y-transformadora/

MEHTA, L. The Scare, Naturalization and Politization of Scarcity. In: MEHTA, L. (editor). The Limits to Scarcity: Contesting the Politics of Allocation. Londres: Earthscan, 2011, p. 13-30.

MC CULLY, P. Silence Rivers: the ecology and politics of large dams. London: Zed Books, 2001.

ORTIZ, A. Las Grandes Obras: Un proyecto político. El caso del represamiento del Paraná Medio en la provincia de Entre Ríos. En: IX Congreso Argentino de Antropología Social. Posadas: Facultad de Humanidades y Ciencias Sociales, Universidad Nacional de Misiones, 2008. Disponible en Internet: http://www.aacademica.org/000-080/272.pdf

RAUSCH, G. Estado, desarrollo y naturaleza: el caso del proyecto Paraná Medio bajo el Paradigma Hidráulico Nacional (Argentina, 1958-1986). Estudios Socioterritoriales, 2016, № 20, p. 11-25.

REBORATTI, C. Ambiente y sociedad. Conceptos y relaciones. Buenos Aires: Ariel, 2000.

ROFMAN, A.; BRUNSTEIN, F.; LAURELLI, E. y VIDAL, A. (coordinadores). Los grandes proyectos y el espacio regional. Presas hidroeléctricas y el sistema decisional. Buenos Aires: CEUR, 1987. 
SANTOS, M. A Natureza do espaço: técnica e tempo, razão e emoção. São Paulo: Hucitec, 2006.

SASSEN, S. Territorio, autoridad, derechos. De los ensamblajes medievales a los ensamblajes globales. Buenos Aires: Katz, 2010.

SCHAPPER, M. Impactos ambientales de los cambios en la estructura exportadora en nueve países de América Latina y el Caribe: 1980-1995. Santiago de Chile: CEPAL, 1999.

SILVEIRA, M.L. Un país, uma região. Fim de século e modernidades na Argentina. São Paulo: FAPESP/LABOPLAN-USP.

SUÁREZ MONTÓRFANO, R. El proyecto hidroeléctrico Corpus Christi. Entre Ríos: Fundación M Biguá/MOTT, 2008. Disponible en Internet:

http://www.mbigua.org. uploads/ElproyectoCorpusChristi ar/.pdf

SVAMPA, M. Movimientos sociales, matrices socio-políticos y nuevos escenarios en América Latina. Kassel: One World Perspective, 2010.

SVAMPA, M. Modelos de desarrollo, cuestión ambiental y giro eco-territorial. En: ALIMONDA, H. (editor). La colonización de la naturaleza. Ecología Política y minería en América Latina. Buenos Aires: CLACSO, 2011, p. 159-192.

SVAMPA, M. Extractivismo neodesarrollista y movimientos sociales. ¿Un giro ecoterritorial hacia nuevas alternativas?. En: GRUPO PERMANENTE DE TRABAJO SOBRE ALTERNATIVAS AL DESARROLLO. Más allá del Desarrollo. Buenos Aires: América Libre, 2012, p. 185-218.

SVAMPA, M. y VIALE, E. Maldesarrollo. La Argentina del extractivismo y el despojo. Buenos Aires: Katz, 2014.

SWYNGEDOUW, E. Liquid Power. Contested Hydro-Modernities in Twentieth-Century Spain. Cambridge: The MIT Press, 2015.

WITTFOGEL, K. Oriental Despotism: A Comparative Study of Total Power. New Haven: Yale University Press, 1957.

WORLD COMISSION ON DAMS. Dams and Development. A new framework for decision-making. London: Earthscan, 2000.

WORSTER, D. Rivers of Empire. Water, Aridity, and the Growth of the American West. New York / Oxford: Oxford University Press, 1985.

WORSTER, D. The Flow of Empire. Comparing Water Control in China and the United States. München: Rachel Carson Center Perspectives/Deutsches Museum, 2011. 